\title{
Quantitative Analysis of the Speed/Accuracy Trade-Off in Transaction Level Modeling
}

\author{
GUNAR SCHIRNER and RAINER DÖMER \\ University of California, Irvine
}

The increasing complexity of embedded systems requires modeling at higher levels of abstraction. Transaction level modeling (TLM) has been proposed to abstract communication for high-speed system simulation and rapid design space exploration. Although being widely accepted for its high performance and efficiency, TLM often exhibits a significant loss in model accuracy.

In this article, we systematically analyze and quantify the speed/accuracy trade-off in TLM. To this end, we provide a classification of TLM abstraction levels based on model granularity and define appropriate metrics and test setups to quantitatively measure and compare the performance and accuracy of such models.

Addressing several classes of embedded communication protocols, we apply our analysis to three common bus architectures, the industry-standard AMBA advanced high-performance bus (AHB) as an on-chip parallel bus, the controller area network (CAN) as an off-chip serial bus, and the Motorola ColdFire Master Bus as an example for a custom embedded processor bus.

Based on the analysis of these individual busses, we then generalize our results for a broader conclusion. The general TLM trade-off offers gains of up to four orders of magnitude in simulation speed, generally however, at the price of low accuracy. We conclude further that model granularity is the key to efficient TLM abstraction, and we identify conditions for accuracy of abstract models. As a result, this article provides general guidelines that allow the system designer to navigate the TLM trade-off effectively and choose the most suitable model for the given application with fast and accurate results.

Categories and Subject Descriptors: I.6.4 [Simulation and Modeling]: Model Validation and Analysis

General Terms: Design

Additional Key Words and Phrases: System level design, transaction level modeling, system-onchip

ACM Reference Format:

Schirner, G. and Dömer, R. 2008. Quantitative analysis of the speed/accuracy trade-off in transaction level modeling. ACM Trans. Embedd. Comput. Syst. 8, 1, Article 4 (December 2008), 29 pages. DOI $=10.1145 / 1457246.1457250 \mathrm{http}: / /$ doi.acm.org/10.1145/1457246.1457250

Author's addresses: G. Schirner and R. Dömer, Center for Embedded Computer Systems, UC Irvine, Irvine, CA 92697-2625, USA; email: \{hschirne, doemer\}@uci.edu.

Permission to make digital or hard copies of part or all of this work for personal or classroom use is granted without fee provided that copies are not made or distributed for profit or direct commercial advantage and that copies show this notice on the first page or initial screen of a display along with the full citation. Copyrights for components of this work owned by others than ACM must be honored. Abstracting with credit is permitted. To copy otherwise, to republish, to post on servers, to redistribute to lists, or to use any component of this work in other works requires prior specific permission and/or a fee. Permissions may be requested from Publications Dept., ACM, Inc., 2 Penn Plaza, Suite 701, New York, NY 10121-0701 USA, fax +1 (212) 869-0481, or permissions@acm.org. (C) 2008 ACM 1539-9087/2008/12-ART4 \$5.00 DOI 10.1145/1457246.1457250 http://doi.acm.org/ $10.1145 / 1457246.1457250$

ACM Transactions on Embedded Computing Systems, Vol. 8, No. 1, Article 4, Publication date: December 2008. 


\section{INTRODUCTION}

Designing embedded systems and system-on-chip (SoC) becomes increasingly challenging. The design space to be explored grows with increasing complexity, while at the same time shorter product life cycles require a shorter time-tomarket. Addressing this gap has been the aim of system-level research. As one main approach, abstract models have been introduced to tackle the design complexity. For one, abstract models exhibit tremendous gains in simulation speed, allowing fast validation and extensive design space exploration.

For communication in particular, transaction level modeling (TLM) has been proposed [Grötker et al. 2002]. TLM abstracts the communication in a system to whole transactions, abstracting away low-level details about pins, wires, and waveforms [Cai and Gajski 2003]. ${ }^{1}$ This results in models that execute dramatically faster than synthesizable, bit-accurate models. This benefit, however, usually comes at the price of low accuracy.

\subsection{TLM Trade-off}

In general, TLMs pose a trade-off between an improvement in simulation speed and a loss in accuracy, as illustrated in Figure 1. The trade-off essentially allows models at different degrees of accuracy and speed. However, having both high speed and high accuracy at the same time is typically not possible. High simulation speed is traded in for low accuracy, and a high degree of accuracy comes at the price of low speed. Models with this trade-off fall into the gray area of the diagram. Models in the dark area are obviously existent, but practically unusable, whereas models in the white area are highly desirable but typically not achievable. Although TLM has been generally accepted as one solution to tackle SoC design complexity, the TLM trade-off, however, has not been examined in detail.

\subsection{Problem Statement}

For this work, we define the problem as follows: a quantitative analysis of the trade-off between simulation performance and accuracy is needed. Definitions of generic metrics and a test framework, applicable to a range of communication protocols, are required to perform the analysis. The immediate analysis goal is to confirm the existence of the TLM trade-off. As a broader goal, we also expect conclusions on (a) classification of abstraction levels, (b) guidelines for model designers in efficiently abstracting communication, and (c) guidance of communication model users in the selection of a suitable model for a given design task.

\subsection{Overview}

In this article, we systematically study and analyze the TLM trade-off quantitatively. More specifically, we quantify the performance gains of TLM and measure the loss in accuracy for a wide range of bus systems. For our

\footnotetext{
${ }^{1}$ General modeling of SoCs consists of two parts, computation and communication. In this article, we focus only on modeling of the communication.
}

ACM Transactions on Embedded Computing Systems, Vol. 8, No. 1, Article 4, Publication date: December 2008. 


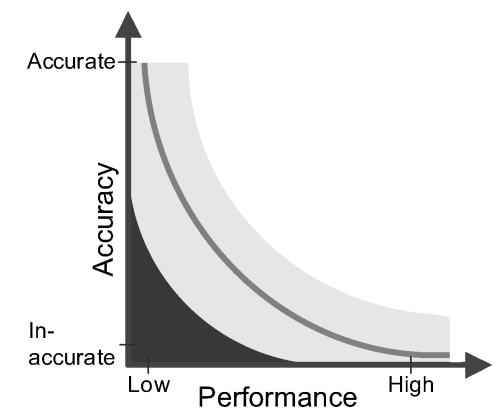

Fig. 1. Transaction level modeling trade-off.

analysis, in Section 4, we define proper metrics and test setups for measuring the performance improvement and the accuracy loss. For each bus system, we use models at different abstraction levels, as defined in Section 3. In particular, we use two classes of TLMs (arbitrated transaction level model [ATLM] and TLM), and compare them against a fully accurate bus functional model (BFM) as a reference.

Our measurements are based on examples from three different bus categories. In Section 5, we analyze the advanced high-performance bus (AHB) of AMBA [ARM 1999], as a representative of parallel on-chip bus systems with centralized arbitration and multiplexed interconnection scheme. AMBA is a widely used and industry-accepted standard for on-chip bus systems. For the second category of off-chip serial busses with distributed arbitration, we investigate the controller area network (CAN) [Bosch 1991] in Section 6. This bus system dominates in automotive applications. Third, in Section 7 , we analyze the category of custom processor-specific busses that are typically much simpler than the general purpose standard busses. Here, we have chosen the Motorola ColdFire Master Bus [Motorola 1997] that is used by the popular ColdFire MCF5206 processor.

The analysis of the first two busses is based on our previous work with the AMBA in [Schirner and Dömer 2006] and CAN in [Schirner and Dömer 2005a]. Whereas these previous publications focused on each bus individually, we will, in this article, combine and generalize the results for different categories and include the ColdFire Master Bus as an example of a third category. Most importantly, based on the results of this range of examples, we will then generalize our TLM analysis in Section 8 and derive general conclusions in Section 9.

\section{RELATED WORK}

System level modeling has become an important research area that aims to improve the SoC design process and its productivity. Languages for capturing SoC models have been developed, examples are SystemC [Grötker et al. 2002] and SpecC [Gajski et al. 2000]. Using TLM [Grötker et al. 2002] for capturing and designing communication architectures has received much attention. Cai and Gajski [2003] provide an initial taxonomy of TLM. [Rose et al. 2005] define a standard for transaction level modeling in SystemC. 
Sgroi et al. [2001] address the SoC communication with a network-on-chip approach. Here, communication is partitioned into layers following the OSI structure. Software reuse is promoted with an increase of abstraction from the underlying communication. Siegmund and Müller [2001] describe with SystemC $\mathrm{C}^{S V}$ an extension to SystemC and propose SoC modeling at three different levels of abstraction: physical description at RTL, a more abstract model for individual messages, and a most abstract model utilizing transactions. Brem and Müller [2003] describe how the CAN bus is modeled using SystemC ${ }^{S V}$. The work also shows the three abstraction levels, but does not give any experimental results on performance or accuracy.

In Caldari et al. [2003] describe the results of capturing the AMBA rev. 2.0 bus standard in SystemC. The bus system has been modeled at two levels of abstraction, first a bus functional model on RTL level, and second a model on TLM level. Their TLM reached a speedup of 100 over the RTL model. Coppola et al. [2003] also propose abstract communication modeling. They present the IPSIM framework and show its efficient simulation. Gerstlauer et al. [2005] describe a layered approach and propose models that implement an increasing number of ISO OSI layers [ISO 1994]. Simulation speedup up to $100 \times$ is shown, but the accuracy analysis is limited.

Haverinen et al. [2002] describe in a white paper three TLMs with increasing abstraction for the OCP-IP protocol. Only their most detailed TL-1 is cycle accurate. They do not show an accuracy analysis for the more abstract models. Pasricha et al. [2004] describe an approach using transaction-based abstraction. Their paper introduces the concept of a model that is cycle count accurate at transaction boundaries (CCATB). This also takes advantage of the limited observability of a transaction to increase simulation performance. However, only a very limited speedup of $55 \%$ over the bus functional model is reported. ${ }^{2}$

\section{TRANSACTION LEVEL MODELING}

TLM allows a wide variety of modeling styles and abstractions. It is not clearly defined in the literature, as also stated by Cai and Gajski [2003] in their approach of structuring the models.

For our modeling, we focus on the granularity of data and arbitration handling. We define three classes of granularity applicable to any bus protocol, and match these granularity classes to three model types. Figure 2 shows the granularity levels with respect to time and indicates the correlation to models and layers.

A user transaction is the most coarse grain element of transferring a contiguous block of bytes with arbitrary length. It is split into bus transactions, which are bus transfer primitives, such as a word transfer. Each bus transaction is usually processed in several bus cycles, which represent the finest granularity in our modeling.

Our classes of granularity follow the layers defined in the ISO OSI reference model [ISO 1994]: the media access control (MAC), the protocol sublayer, and

\footnotetext{
${ }^{2}$ Our results show a speedup of up to four orders of magnitude. 


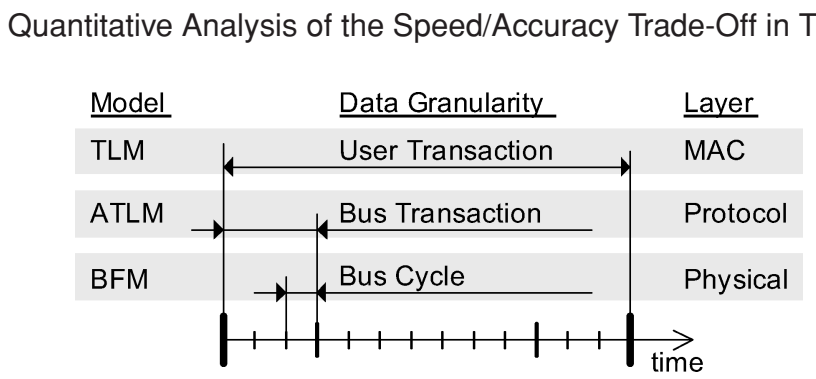

Fig. 2. Model classes and their granularity.

the physical layer. Each considered layer handles data and arbitration at its own granularity.

The media access layer provides a transmission service for a user transaction, which is a contiguous block of bytes. This layer divides the arbitrarily sized user transaction into smaller bus transactions observing the bus addressing rules, and transfers these byte blocks using the protocol layer. The protocol layer transfers data as bus transactions, which are bus primitives (e.g., bytes, words, or 4-word burst). It in turn uses the physical layer, which provides services to sample and drive individual bus wires at bus cycle granularity.

For dealing with the description complexity, we chose a layered architecture for our bus models similar to Gerstlauer et al. [2005]. Using a system level modeling approach, each layer can be implemented as a separate channel using a system-level design language (SLDL). ${ }^{3}$ Then, different models can be easily created by composing the channels hierarchically.

According to our classes of granularity, we consider models at three different abstraction levels; the TLM that models user transactions, the ATLM at bus transaction granularity, and the bus cycle accurate BFM.

\subsection{Transaction Level Model}

The TLM ${ }^{4}$ is the most abstract model; it only implements the media access layer. The user data, handled at the user transaction granularity, is transferred regardless of its size in one chunk using a single memcpy. Timing is simulated by a single wait-for-time statement, covering the whole user transaction. Arbitration is not modeled. Instead, concurrent access is resolved using a semaphore once per user transaction. The semaphore-based contention resolution depends on the simulation environment and is independent of the arbitration of the actual bus protocol.

\subsection{Arbitrated Transaction Level Model}

The ATLM simulates the bus access with bus transaction granularity (e.g., AHB bus primitives). It uses the MAC layer to split user transactions into bus transactions and implements an own abstracted protocol layer. The ATLM

\footnotetext{
${ }^{3}$ We have used SpecC [Gajski et al. 2000] as the SLDL of choice, we could have used SystemC just as well.

${ }^{4}$ In the general, sense we consider TLM to be a class of models and our bus models are a part of this class. To be consistent with our previous publications, however, we have kept TLM also as a name of our most abstract model.
}

ACM Transactions on Embedded Computing Systems, Vol. 8, No. 1, Article 4, Publication date: December 2008. 
accurately models the bus arbitration for each bus transaction. We implement the arbitration and the bus without an own flow of execution to maximize simulation performance. Although this model correctly models arbitration, it is not pin-accurate and not cycle-accurate in all cases.

\subsection{Bus Functional Model}

The BFM is a synthesizable, bus cycle-accurate and pin-accurate bus model. It implements all layers down to the physical layer and covers all timing and functional properties of the bus definition. It handles arbitration per bus transaction and has the capability to take arbitration decisions on a bus cycle granularity. This model may include additional hardware, such as an arbiter, to correctly implement the bus standard.

\subsection{Comparison with Other TLM Abstractions}

In order to relate our TLM abstraction levels to other abstraction schemes, we will briefly compare our models to the OCP-IP and and SystemC TLM standards.

In comparison to the OCP-IP TLM definitions [Haverinen et al. 2002], our models range between TL0 and TL3. Our BFM correlates most closely to TL0, since it models all bus wires, implements active bus components if applicable (e.g., multiplexers of AHB) and is based on an explicit clock. Our ATLM compares to TL2, since it uses bus transactions and is cycle-approximate in the general case. ${ }^{5}$ Our TLM bus model lies between TL2 and TL3. It is more abstract than TL2, since it is based on user transactions (messages in the OCPIP). On the other hand, it provides cycle-approximate timing, hence it is more detailed than TL3.

A comparison with the SystemC TLM standard [Rose et al. 2005] is more difficult, because the version 1.0 does not define the SystemC abstraction levels in detail. Our BFM matches the cycle callable (CC) model, since it is cycleaccurate and bit-accurate (CABA). In addition to the CC properties, our BFM is pin-accurate (PA) as well. Both our ATLM and the TLM could be called a programmers view + time (PVT) model, since both provide cycle-approximate (CX) timing.

\section{METRICS AND MEASUREMENT SETUP}

Given the model classification, we will now describe our metrics and setup for analyzing our models. We focus on two aspects. First, we look at the simulation performance, since a performance gain is the main premise of TLM. Second, we evaluate the timing accuracy.

\subsection{Performance}

Our metric for the performance is the simulation bandwidth. The bandwidth is the amount of data transferred over the simulated bus in 1 second of real-time.

\footnotetext{
${ }^{5}$ The ATLM can be cycle-accurate for protocols without preemption, as we will show later. 


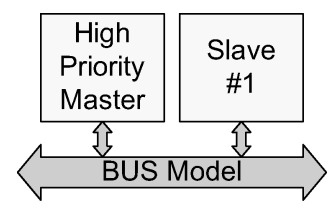

Fig. 3. Single master setup for performance measurements.

We have measured the simulation performance of each model in a minimal scenario with one master and one slave (Figure 3). A transaction with random content is performed repeatedly, without any delay in between. We have measured the simulation time (also referred to as real time or wall clock time) over a set of repeated iterations (e.g., 5,000) and computed the simulation bandwidth. In order to estimate the scalability, we repeat this test for an increasing size of the user transaction.

Besides the data transfer and the controlling loop, the test masters and slaves do not execute any other code. This minimizes their impact on the overall simulation time and allows us to examine solely the performance of the bus models. All tests have been executed using the SpecC V 2.2.0 discrete event simulator using Quick Threads on a Pentium $4(2.8 \mathrm{GHz})$ PC running Red Hat Enterprise WS 3.

\subsection{Accuracy}

Accuracy can be segregated into many aspects. For the purpose of this article, we will distinguish three aspects of accuracy: functionality, represented feature detail, and timing. ${ }^{6}$ For our analysis, we will fix the first, vary the second, and measure the third aspect.

The first aspect of functional accuracy requires that the data transmitted by the sender reaches correctly the receiver. Since this is a necessary requirement for a functional simulation, all our models are functionally accurate. The second aspect is the represented detail level, which states how many features of the bus are actually present in the model. We vary this aspect in our model under test, ranging from accurately modeled pins and waveforms in the BFM up to a single memcpy and wait-for-time statement in the TLM. ${ }^{7}$ The third aspect is the accuracy in timing which we measure in our tests. To compare the models, we use the timing of user transactions.

4.2.1 Timing Accuracy Metrics. The relevant measurements for expressing the accuracy of a model depend on the prediction goal of the simulation. We have identified two goals: First, the prediction of the application latency due to an individual bus access, and second, the prediction of the application finish time. For these goals, we use two metrics. First, by analyzing the individual duration of a user transaction, the application latency due to a bus access

${ }^{6}$ We omit one dimension of model accuracy, the "coding quality," due to the difficulty of a realistic measurement. In order to keep this factor as constant as possible, all models have been implemented with the same optimization effort by the first author.

${ }^{7} \mathrm{~A}$ detailed table of represented features is given later in Section 6 for the CAN bus. 


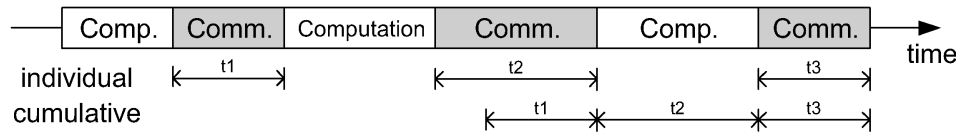

Fig. 4. Cumulative and individual transfer time.

can be inferred. Second, the cumulative duration, which is the sum of all user transactions, shows how much the application finish time is delayed by the performed communication. For our tests, we prefer the cumulative transfer time over the actual finish time, since the latter includes the constant computation time between transactions (simulated by a delay), which is independent of the utilized bus model.

Figure 4 shows an example of a bus node's activity over time which progresses along the x-axis. Any process alternatively performs computation or communication, the latter of which we are interested in. For the individual duration, we analyze the transfers $t_{1}, t_{2}$ and $t_{3}$ separately. For the cumulative analysis, we use the sum of the three transfers.

For the purpose of analyzing the accuracy based on the individual and cumulative duration, we define for this article the duration error as the percentage error over the bus standard ${ }^{8}$ :

$$
\begin{aligned}
d_{\text {std }}: & \text { duration as per standard } \\
d_{\text {test }}: & \text { duration in model under test } \\
\text { error }_{i}= & 100 * \frac{\left|d_{\text {test }}-d_{\text {std }}\right|}{d_{\text {std }}}
\end{aligned}
$$

Given this error definition, a timing accurate model exhibits $0 \%$ error. Note also that a particular model may have an error of more than $100 \%$ (i.e., the model under test shows more than twice the simulated time).

4.2.2 Bus Contention Metrics. Our models differ in the granularity of data and arbitration handling. We, therefore, expect a significant correlation between bus contention and accuracy. Thus, we use contention as an input to our measurements.

For our tests, we examine the status of a user transaction to determine the bus contention. This makes the contention definition independent of the actual bus implementation and can be applied to different bus systems. For this article, we define the bus contention as the percentage overlap between user transactions:

$$
\text { contention }=100 * \frac{\text { bus cycles with two or more active user transactions }}{\text { bus cycles with at least one active user transaction }}
$$

Figure 5 depicts example contentions of $0 \%, 25 \%$ and $50 \%$. The low-priority transaction starts delayed due to an ongoing high-priority transaction, which is considered bus contention in our definition.

${ }^{8}$ For practical purposes, we use a two-step approach. We first implement and validate the BFM according to the bus standard. Then, we use the BFM to obtain the reference timing.

ACM Transactions on Embedded Computing Systems, Vol. 8, No. 1, Article 4, Publication date: December 2008. 


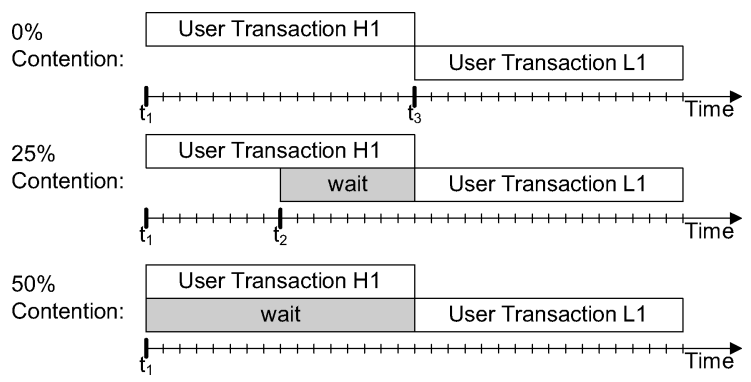

Fig. 5. Bus contention.

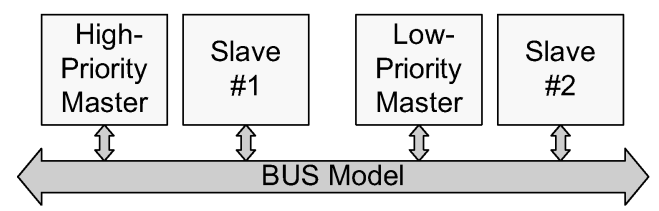

Fig. 6. Dual master setup for accuracy measurements.

4.2.3 Accuracy Measurement Setup. The actual timing accuracy highly depends on the test setup and the communication patterns of the involved applications. Therefore, we define a generic test setup and a procedure that covers a range of applications, so that the designer can derive the expected accuracy for her/his particular setup.

We use a generic test setup with two masters and two slaves connected to the same bus, as shown in Figure 6. Each master transfers a predefined set of 5,000 user transactions to a slave target. Each transaction varies linear randomly in the base address, size ( 1 to 100 bytes), content, ${ }^{9}$ and the delay to the next transaction. This delay simulates the application's computation. It ranges from a zero delay to a maximum delay value that depends on the desired contention (see later discussion). The list of all transactions is created before the actual test by using a random number generator with a fixed start seed.

During the simulation, the start time and the duration is recorded for each individual user transaction and each master. The test is repeated for each implemented bus model. Since each model transfers the same set of user transactions, the results are comparable and can be analyzed together.

We have repeated the described test for different levels of bus contention. Since the bus contention cannot be controlled directly, we have instead varied the maximum delay between user transactions for each test run. This results in a varying bus utilization per master. Since the two masters share the same bus in our setup, the utilization correlates to the amount of bus contention. We have measured the resulting amount of bus contention according to our previous definition using the BFM, by checking for each clock cycle whether one or two user transactions are active. After we have determined the maximum

${ }^{9}$ Please note that for some protocols (e.g., the CAN protocol) the content of the transaction can influence the transaction duration. 


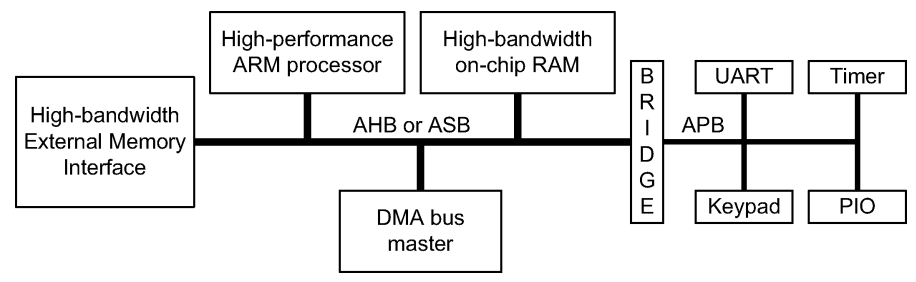

Fig. 7. AMBA bus architecture (Source: ARM [1999]).

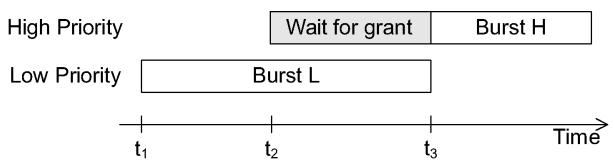

(a) Locked transfer

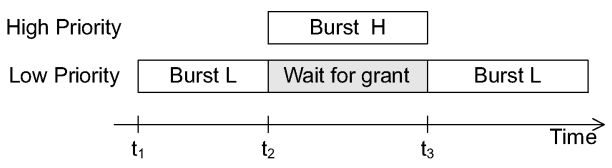

(b) Unlocked transfer

Fig. 8. AMBA AHB operation modes.

delay that produces a desired contention, we use the created transaction set for measuring the abstract models.

\section{AMBA}

The first bus system is an example for an on-chip bus system with a centralized arbitration scheme [Schirner and Dömer 2006]. We use the advanced microprocessor bus architecture (AMBA) for our analysis.

\subsection{Introduction}

AMBA defined by ARM [ARM 1999] is a widely used and industry accepted standard for an on-chip bus system. The AMBA standard contains a group of busses, which are used hierarchically, as shown in Figure 7. We focus on the advanced high-performance bus (AHB), a system bus designed for connecting high-speed components including ARM processors.

The AHB is a multimaster bus that operates on a single clock edge and employs a centralized arbitration scheme. High performance is achieved by a pipelined operation that overlaps address and data phases, as well as by the usage of burst transfers. Split and retry transfers allow the slave to free the bus if the requested data is temporarily unavailable. The AHB uses a multiplexed interconnection scheme to avoid tri-state drivers.

5.1.1 Locked versus Unlocked Transfers. The AHB allows two operation modes, locked and unlocked transfers. These differ in whether preemption of a burst transfer is allowed. An example is shown in Figure 8.

In the locked mode in Figure 8(a), a ongoing burst cannot be preempted by a higher-priority bus transaction. The high-priority transaction released at $t_{2}$ is delayed until the current low priority bus transaction finishes at $t_{3}$. In the unlocked mode in Figure 8(b), the high-priority transaction preempts the ongoing low priority bus transaction and gains bus access already at $t_{2}$. Since the operation mode influences the granularity of the arbitration decision, we will analyze these two operation modes separately. 


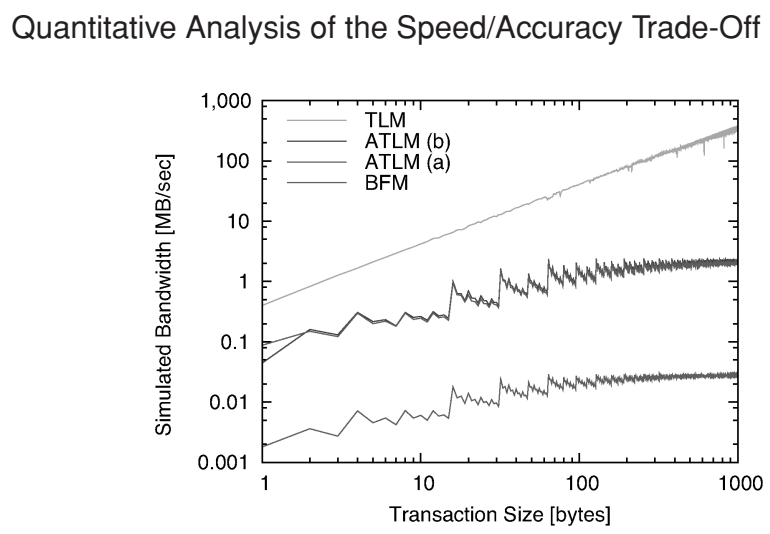

Fig. 9. Performance for the AMBA AHB models.

\subsection{Models}

For modeling the AMBA AHB, we have applied the granularity-based approach described in Section 3.

Transaction Level Model. The TLM is the most abstract model that operates on the granularity of user transactions. It resolves contention independent of the priority arbitration using a semaphore once per user transaction, as described in Section 3.1. It provides basic support for bursts.

Arbitrated Transaction Level Model. The ATLM operates on the bus transaction granularity, such as a StoreWord, StoreByte, LoadBurst. It adds support for priority-based arbitration and reflects the effects of pipelined operation. We have implemented two variants of the ATLM that differ in the time frame to collect arbitration requests. On an idle bus, the ATLM (a) collects requests for one clock cycle before making a decision, as required by the standard. The ATLM (b), on the other hand, makes the decision immediately after receiving the first request. Both variants behave identical when the bus is busy: requests are collected while a bus transaction is active, and the highest priority master continues after that.

Bus Functional Model. The BFM is the bus-cycle accurate and pin-accurate bus model. In order to correctly model the bus architecture, we have implemented several active components, such as multiplexers, an arbiter and an address decoder. On top of the features offered by the ATLM, the BFM supports unlocked (preemptable) transfers. At this point, our model does not support split and retry operations.

We have functionally validated all described models. We have also validated the cycle count timing of the BFM against the standard for all bus primitives, and have compared the waveforms with examples in ARM [1999, 2003]. Finally, we have ensured that all abstract models show the correct timing in the single master setup.

\subsection{Performance Analysis}

Our performance measurements (Figure 9) confirm the TLM expectations: The simulation speed increases significantly with abstraction. The performance 
Table I. Performance Comparison for Transferring 512 Bytes Using AMBA AHB Models

\begin{tabular}{|l||c|c|c|c|}
\hline & BFM & ATLM (a) & ATLM (b) & TLM \\
\hline \hline Simulation Time [ms] & 16.75 & 0.2137 & 0.206 & 0.00246 \\
\hline Sim. Bandwidth [MB/sec] & 0.03 & 2.29 & 2.37 & 198 \\
\hline Rel. Speedup over BFM & 1 & 78 & 81 & 6802 \\
\hline
\end{tabular}

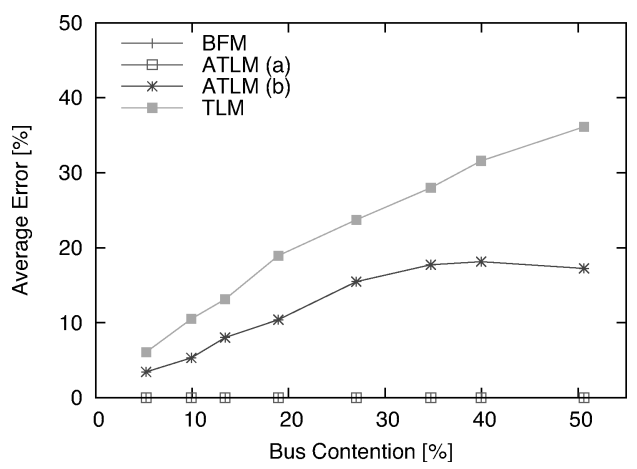

Fig. 10. Individual timing accuracy of locked transfers for the AMBA AHB models.

raises with each TLM abstraction by two orders of magnitude. However, no significant performance difference exists between the variants within the ATLMs. The additional abstraction of the (b) variant does not yield a significant speed improvement. Table I compares the performance in detail for transferring 512 bytes.

The TLM executes the fastest among the analyzed models. Its simulation bandwidth increases linearly with the transaction size, since a constant number of operations is executed for each transfer (one memcpy and one wait-fortime). The ATLMs are two orders of magnitude slower due to the finer granularity of modeling individual bus transactions. Starting with the ATLMs, the graphs exhibit a saw tooth shape due to the nonlinear split of user transactions into bus transactions (e.g., 3 bytes are transferred in 2 bus transactions: byte + short, whereas 4 bytes are transferred in 1 bus transaction: a word). The BFM is again two orders of magnitude slower than the ATLMs due to the fine grain modeling of individual wires and additional active components (e.g., multiplexers).

\subsection{Accuracy Analysis}

The performance analysis for the AHB has shown the impressive speed benefits of TLM. Now we will evaluate the accuracy reduction, that the designer has to accept for achieving the higher simulation speed. As described before, we analyze locked and unlocked transfers separately.

5.4.1 Analysis for Locked Transfers. With locked transfers, a burst cannot be preempted by a higher priority master. Figure 10 shows the average timing error over a range of bus contention for the high priority master. The x-axis 


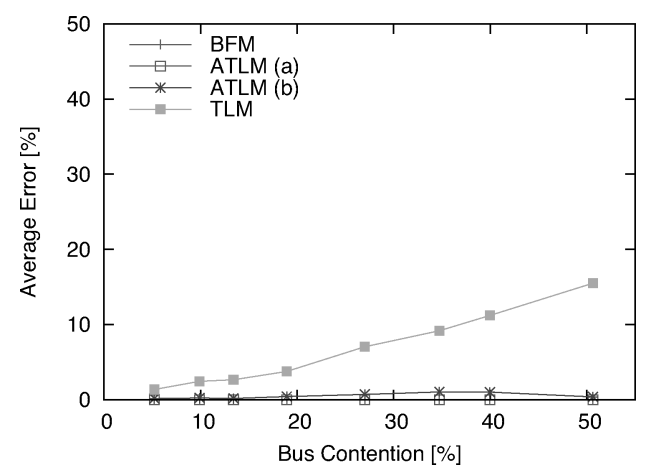

Fig. 11. Cumulative timing accuracy of locked transfers for the AMBA AHB.

denotes the amount of bus contention in percent, each measurement point for a model reflects the average error over the analyzed 5,000 user transactions.

The BFM and the ATLM (a) perform accurately over the whole range of bus contention (their graphs lie on top of the x-axis). Since the test setup uses only locked transfers, a burst is not preemptable and therefore no arbitration test is needed within a bus transaction. The features abstracted away in the ATLM (a) are not exercised. The ATLM (b), which makes an immediate decision and does not collect further requests, shows an inaccuracy of up to $18 \%$. It may mispredict bus access when the two masters attempt a bus access within the same clock cycle.

On the other hand, the ATLM (b) is accurate, when the additional bus request arrives during an active bus transaction. Therefore, the error rate plateaus for bus contentions higher than $35 \%$.

The TLM, which handles contention resolution on user transaction level with a semaphore, performs the least accurate due to the coarse grained decision independent of the master's priority. Its inaccuracy amounts up to $35 \%$.

The measurements show very similar results for the low priority master. Hence, its graph is omitted for brevity, but can be found in Schirner and Dömer [2005b].

To predict the delay of an application due to bus communication, we have also analyzed the same experimental data in terms of cumulative transfer time. Figure 11 shows the error in the cumulative duration for the high priority master using locked transfers. ${ }^{10}$

As in the previous graph, the lines for both BFM and ATLM (a) lie on top of the x-axis. The graph reveals that the mispredictions made by the less accurate ATLM (b) do average out. Both ATLM variants are good predictions for the application finish time. Only the mispredictions of the TLM do not average out, its error increases linearly to $15 \%$ for $50 \%$ bus contention.

5.4.2 Analysis for Unlocked Transfers. We have repeated the same experiment for unlocked transfers. A burst may be preempted in this operation mode by a higher priority bus master, to be resumed later. As before, we have

${ }^{10}$ The graph for the low priority master is omitted, due to its similarity. 


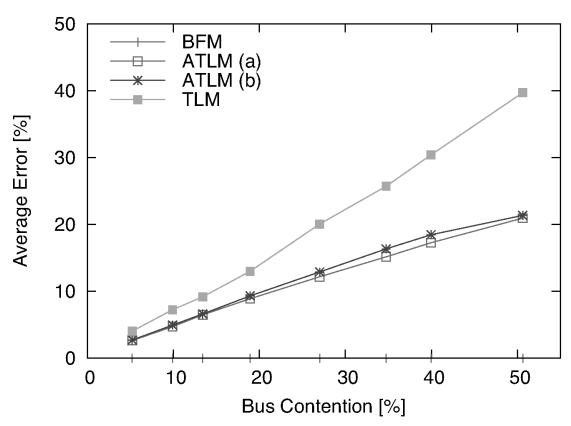

(a) High-priority master

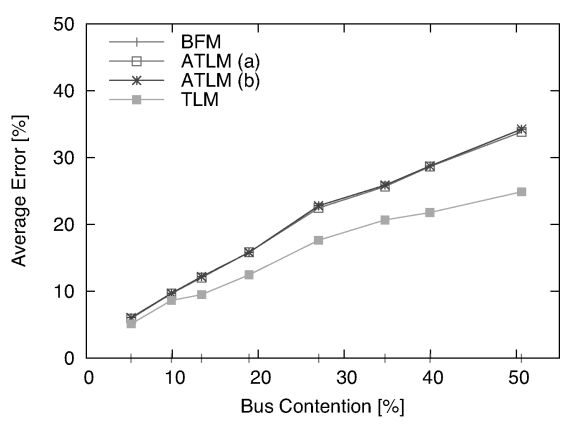

(b) Low-priority master

Fig. 12. Cumulative timing accuracy for unlocked transfers for the AMBA AHB.

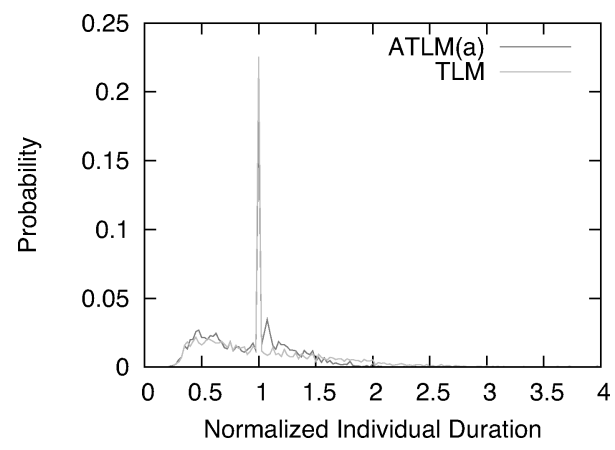

Fig. 13. Histogram of normalized transaction duration.

analyzed the accuracy for both: the individual transfer duration and the cumulative transfer time. Both analysis aspects yield similar results; therefore, only one-the accuracy based on cumulative transfer time-is shown in Figure 12. Since the results differ by priority, the graphs for the high-priority master and the low-priority master are shown side by side.

Figure 12 shows that only the BFM yields accurate results. With unlocked transfers, an arbitration decision is also necessary within a bus transaction. Therefore, the ATLM (a) does now show an error of up to $35 \%$ for the lowpriority master. It handles arbitration only at the bus transaction granularity. The difference between the variants of the ATLM becomes insignificant. The ATLMs perform similar for the high and the low priority master.

The TLM can only give a very rough timing estimate. It exhibits a linear increasing error of up to $45 \%$ for the high-priority master; the test requires arbitration handling for each bus cycle. The error is less for the lower-priority master.

5.4.3 Error Distribution. Until now, our analysis has focused on the average of errors, which is applicable when predicting the overall system performance. Additionally, the error distribution may be of interest, to judge the range of errors to be expected during simulation. Figure 13 shows a histogram of the transaction durations normalized to the duration in the BFM. A value of 1 


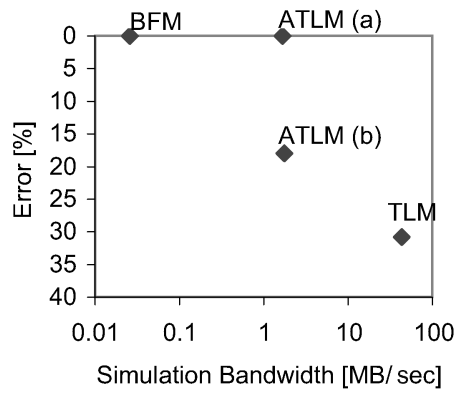

(a) Locked transfers

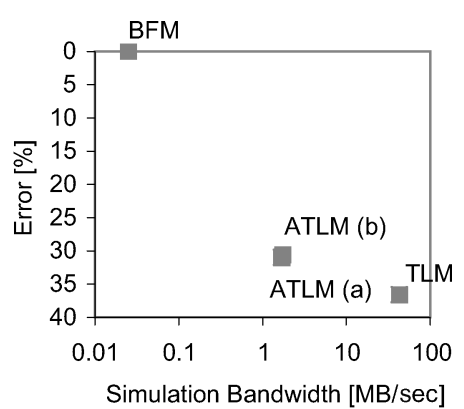

(b) Unlocked transfers

Fig. 14. AMBA AHB TLM trade-off.

indicates an accurate prediction, with a value of 2 the abstract model simulates a transaction with twice the time of the BFM.

For simplicity, we only compare the ATLM (a) and the TLM. Both are timing accurate for about $20 \%$ of the user transactions. The normalized duration of the ATLM range from 0.25 to 2, the results of the TLM are more wide spread, ranging from 0.25 up to 4 . Thus, the ATLM has a tighter error bound.

\subsection{Summary for the AMBA AHB}

Figure 14 summarizes our analysis for the AMBA AHB and depicts the actual TLM trade-off. It shows the detailed relation between performance and accuracy. The $\mathrm{x}$-axis denotes the performance in simulation bandwidth for transferring a 100 byte user transaction. The y-axis denotes the accuracy as the average error in individual timing at $40 \%$ bus contention for the low-priority master.

Compared with Figure 1, Figure 14 show the expected trade-off curve, with the exception of the ATLM (a) in Figure 14(a), which shows medium bandwidth at $0 \%$ error due to the locked transfer mode. Note that in Figure 14(b), the ATLM (a) does not exhibit this exception, lying directly over the ATLM (b).

In summary, our measurements of the AMBA AHB confirm the expectation of a significant speedup with abstraction. The ATLMs are two orders of magnitude faster than the BFM and simulate with more than 1MB. Decreasing the granularity further to user transactions, as done by the TLM, increases the speed for another two orders of magnitude.

Both variants of the ATLM show the same performance. This indicates that staying within the same granularity level, in this case bus transactions, usually leads to similar performance.

The increasing abstraction, however, also leads to a decreased timing accuracy. The BFM is the slowest model, but always delivers accurate results. The medium speed ATLM model, is accurate for the locked transfers, but is inaccurate for the unlocked transfers (30\% error). The fastest model, the TLM, also is most inaccurate (30\% error for locked and $37 \%$ for unlocked transfers).

The ATLM (b) variant, which performs a reduced arbitration, is not efficient. Although it is not faster than the ATLM(a) it does produce inaccurate results even for the locked transfers (17\% error). 
Table II. AMBA AHB Model Selection

\begin{tabular}{|l|c|c|}
\hline Environment Condition & Model & Speedup \\
\hline $\begin{array}{l}\text { - Single master bus } \\
\text { - No bus contention }\end{array}$ & TLM & $10^{4}$ \\
\hline $\begin{array}{l}\text { - Only locked transfers } \\
\text { - Unlocked transfers, low contention }\end{array}$ & ATLM & $10^{2}$ \\
\hline - Unlocked transfers, high contention & BFM & $10^{0}$ \\
\hline
\end{tabular}

Based on our performance and accuracy analysis, Table II lists the fastest model that yields acceptable results for a given situation and simulation focus.

All our AHB models are accurate in case of zero bus contention. Therefore, the TLM should be chosen in an architecture with a single master or when no bus contention is expected. Then, it delivers accurate results the fastest. A system that only uses locked transfers can be accurately simulated by the ATLM. Its results are also acceptable for unlocked transfers in systems with a low bus contention. However, when simulating unlocked transfers under high bus contention only the BFM yields accurate results.

We have modeled the AMBA AHB as a representative for the category of on-chip parallel busses with centralized arbitration. We expect that our results are a good indicator for the behavior of models of other busses in this category, for example, the IBM CoreConnect Processor Local Bus [IBM 2004].

\section{CAN}

Our second bus example [Schirner and Dömer 2005a] falls into the category of an off-chip serial bus with decentralized arbitration. The Controller Area Network (CAN) is a serial communication protocol introduced by the Robert Bosch GmbH [Bosch 1991] that was designed for automotive applications.

\subsection{Introduction}

CAN is a serial multimaster broadcast bus. Frames, with up to 8 bytes user data, are received by all bus nodes and distinguished by the frame identifier. Each bus node decides using local rules whether or not to process a frame. The frame identifier also serves as a priority. If multiple senders simultaneously attempt a transmission, the collision free CSMA/CA arbitration will guarantee that the highest priority frame will succeed undisturbed.

After transmitting the start of frame bit (see Figure 15), the frame identifier is transmitted with the most significant bit first as a sequence of recessive (1) and dominant (0) bus states. During transmission, each sender compares the sent and received signal. If a sender has sent a recessive bit but detects a dominant bit, it will back off from transmission because another sender must have started a higher-priority frame.

In order to allow detection of corrupted data, each CAN frame includes a 15bit CRC. In case of a CRC mismatch, a retransmission of the frame is triggered. The protocol also defines elaborate error detection and error confinement rules for protection against faulty bus nodes. 


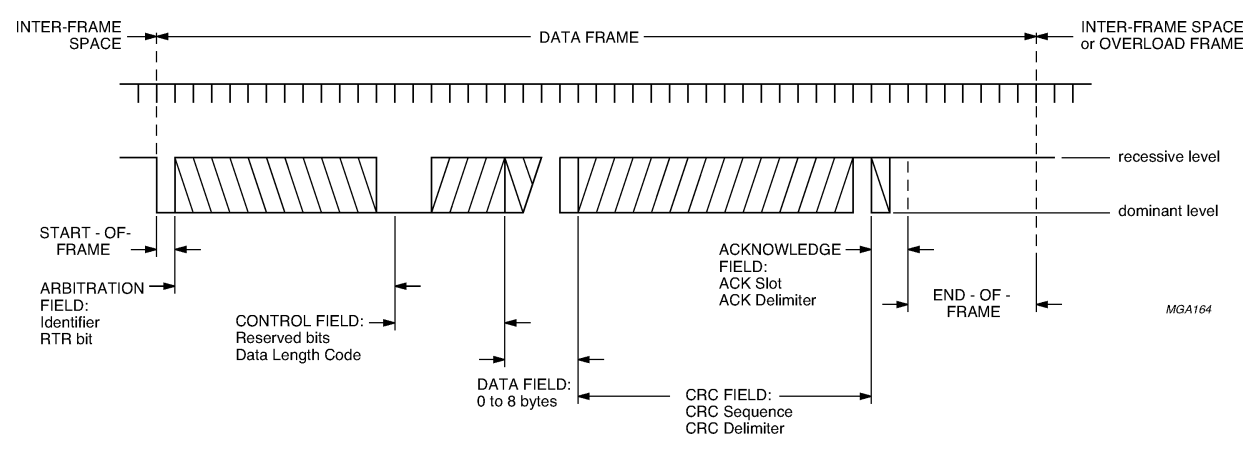

Fig. 15. CAN data frame (Source: Philips).

The CAN serial protocol operates without a centralized clock. Each bus node synchronizes on the bit stream of the sender. A bit-stuffing rule guarantees sufficient edges for this synchronization. After transmitting 5 bits of equal polarity, a bit of opposite polarity is introduced.

For modeling a CAN bus, the following features are candidates for abstraction:

- Serial protocol

-Bit synchronization

-Error detection and confinement

-Bit error detection using a 15-bit CRC

-Bit stuffing

-Arbitration, bus access controlled by CSMA/CA

\subsection{Models}

We have applied our granularity-based abstraction to model the CAN. For each model, we have selected a subset of the above listed features.

Transaction Level Model. The most abstract model is identical to the TLM of the AHB. Contention resolution is implemented on the user transaction granularity by using a semaphore (which ignores the frame identifier).

Arbitrated Transaction Level Model. The ATLM simulates arbitration accurately for each bus transaction (CAN frame) based on the frame identifier. It collects all requests during the start of frame, and proceeds with the highest priority frame.

Again, we have defined two variants for the ATLM model. The ATLM (a) performs a bitwise inspection of the frame in order to calculate the CRC and handle stuff bits: a stuff bit is inserted/removed each time 5 bits of equal polarity are found. Note that due to the bit stuffing, the physical frame length depends on the frame content.

The ATLM (b) does neither calculate the CRC nor does it handle stuff bits. It avoids the costly bit inspection and is expected to execute faster than the ATLM (a), however at the cost of accuracy. 
Table III. Summary of Features Supported or Abstracted Away in the CAN Models

\begin{tabular}{|l||c|c|c|c|}
\hline Feature & BFM & ATLM (a) & ATLM (b) & TLM \\
\hline \hline Serial transmission, bit sync & yes & no & no & no \\
Error detection, confinement & yes & no & no & no \\
CRC calculation, bit stuffing & yes & yes & no & no \\
CSMA/CA arbitration & yes & yes & yes & no \\
\hline
\end{tabular}

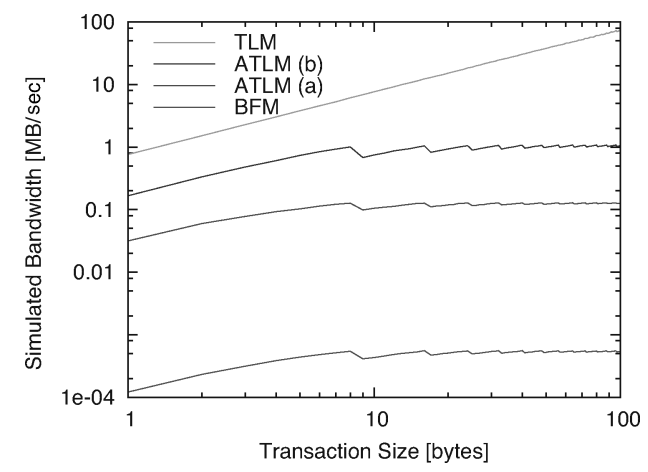

Fig. 16. Performance of the CAN models.

Bus Functional Model. The BFM implements all features of the specification. It protects the data by the $\mathrm{CRC}$, handles stuff bits and performs arbitration. The frame data is sent and received serially and the nodes clock is synchronized to the bit stream according to Bosch [1991] and Hartwich and Bassemir [1999].

Table III summarizes the features included in each model.

\subsection{Performance Analysis}

As with the AMBA AHB, we analyze the CAN models in terms of performance and accuracy. The results of our performance measurements in terms of simulation bandwidth over an increasing user transaction size are shown in Figure 16. In addition, Table IV compares the performance of the models in detail for a user transaction of 16 bytes.

Our performance measurements of the CAN show a similar dramatic increase in simulation speed as seen for the AHB. The TLM shows the highest simulation bandwidth, which increases linearly due to the constant number of operations. It achieves $12 \mathrm{MB} / \mathrm{sec}$ when using 16 byte user transactions.

The ATLM (b) is the next slower model $(12 \times)$. It does not model bit stuffing and CRC. Since the ATLM models individual bus transactions, a step is noticeable in the graph for each 8 bytes-an additional CAN message is needed for transferring the user data. The execution time increases linearly with the amount of bus transactions, reaching a bandwidth of $1 \mathrm{MB} / \mathrm{sec}$.

The ATLM (a) performs 8 times slower than the ATLM (b), since it inspects every bit of the message for the bit stuffing and the CRC calculation. 
Table IV. Performance Comparison for Transferring 16 bytes Using CAN Models

\begin{tabular}{|l||c|c|c|c|}
\hline & BFM & ATLM (a) & ATLM (b) & TLM \\
\hline \hline Simulation Time [ms] & 27.4 & 0.12 & 0.015 & 0.0012 \\
\hline Sim. Bandwidth [MB/sec] & 0.0006 & 0.127 & 1.05 & 12.3 \\
\hline Speedup over BFM & 1 & 228 & 1879 & 22124 \\
\hline Rel. Speedup over previous & - & 228 & 8 & 12 \\
\hline
\end{tabular}

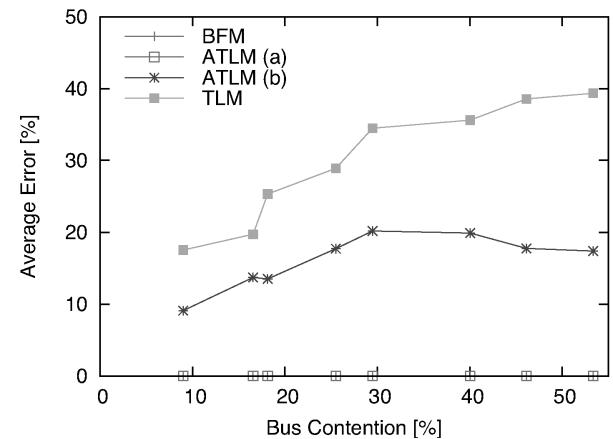

(a) High-priority messages.

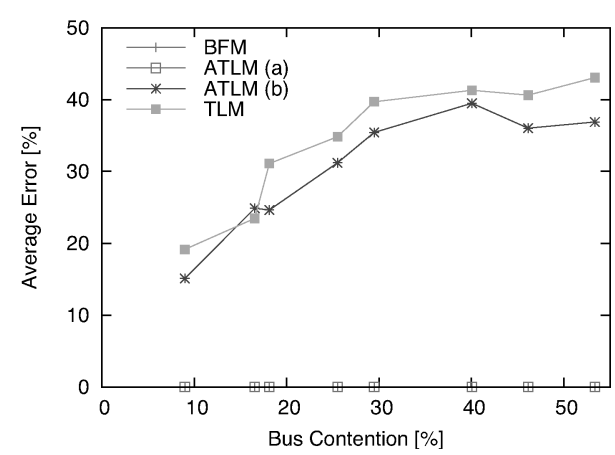

(b) Low-priority messages.

Fig. 17. Individual timing accuracy for the CAN models.

The BFM is another two orders of magnitude slower than the ATLM (a), due to the additional computational effort for the fine grained serial transmission and the bit synchronization. In addition, the structure of the implementation reduces the performance. For each bus node, two extra threads of execution are required, one for the bit stream processor and one for the bit timing logic.

\subsection{Accuracy Analysis}

We use our generic dual master setup (Figure 6) for measuring the CAN model accuracy. However, CAN does not have a master/slave distinction, so we have modified our setup to two nodes acting as senders and two nodes acting as receivers. We let the user transactions vary in message id, length and content of the transaction ( 1 to 16 bytes), and in the delay between two transactions. Each sender uses an exclusive range of message identifiers. One will send messages with high-priority identifiers ( 0 to 511 ), the other emits messages with lowpriority (identifiers 512 to 1,023).

6.4.1 Analysis Based on Individual Transfer Duration. The first set of resulting graphs in Figure 17 show the average individual timing error over an increasing bus contention separately for high- and low-priority messages.

Figure 17(a) shows that the ATLM (a), which includes bit stuffing and CRC calculation, performs as accurate as the BFM (both graphs lie on top of the $\mathrm{x}$-axis). This result has to be interpreted with perspective to the restrictions of the test, which are: no propagation delay between sending and receiving on the CAN bus, all delays between user transactions are multiple of the CAN bit time and the test starts aligned to the bit clock of the first sender. With this setting 


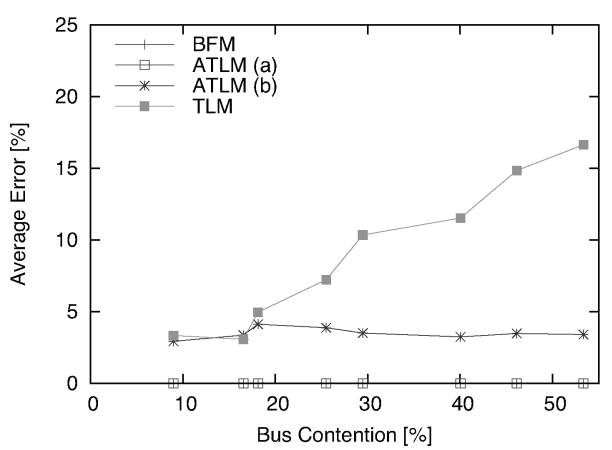

(a) High-priority messages.

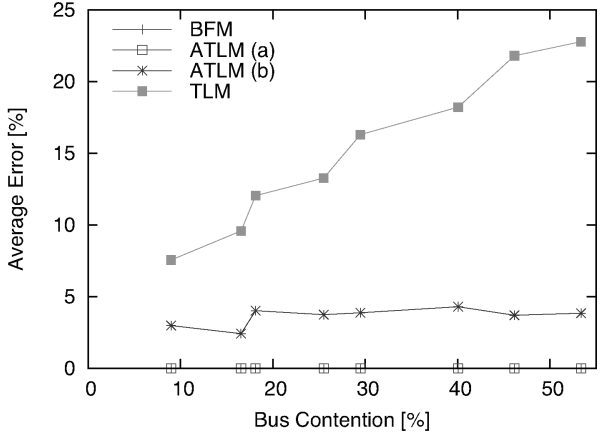

(b) Low-priority messages

Fig. 18. Cumulative timing accuracy for the CAN models.

(reasonable for a simulation environment only) all bus accesses are performed aligned to the CAN bit clock, and no subcycle information is needed. In this situation, the additional capabilities of the BFM (i.e., bit synchronization) are not exercised and both the BFM and the ATLM (a) are accurate.

The ATLM (b), due to the missing bit stuffing and CRC, performs inaccurately. For messages in the high-priority range, the inaccuracy starts with $10 \%$ for low-contention situations and, after linearly rising to $20 \%$ inaccuracy, plateaus at $30 \%$ contention. Without the bit stuff modeling, an individual message transfer is-depending on its content-shorter than in the bus functional model. Therefore, the arbitration interaction between the two senders differs. With an increasing contention the user transactions of the low-priority band increasingly influence the high-priority transactions. However an earlier started low-priority transaction, which may consist of multiple CAN frames, can delay a later started high-priority user transaction only for up to one frame. A second started CAN frame of the low priority transaction will lose arbitration, which leads to the plateau in inaccuracy at $30 \%$. Looking at the same scenario with reversed priorities, this limitation does not apply. A low-priority user transaction may be delayed for a full high-priority transaction consisting of many CAN frames. Hence, the timing error of the ATLM (b) increases without a plateau for the low-priority user transactions (Figure 17(b)) with increasing contention.

The TLM yields uniform results for both the high- and low-priority sender. For both cases, the inaccuracy increases with the bus contention. As expected, the TLM suffers the most in loss of accuracy ( $40 \%$ inaccuracy at $45 \%$ contention).

6.4.2 Analysis Based on Cumulative Transfer Duration. Figure 18 shows the accuracy results based on cumulative transfer time.

The analysis reveals that mispredictions of the ATLM (b) for individual CAN frames average out during the test, since the model correctly captures arbitration. Regardless of priority, a constant error of about $4 \%$ is measured. This can be attributed to not modeling the bit stuffing which in average adds $4 \%$ bits. The TLM, with its coarse grain priority-independent contention resolution, shows for both priority ranges a linear increasing error. 
Table V. CAN Model Selection

\begin{tabular}{|l|c|}
\hline Environment Condition & Applicable Model \\
\hline \hline $\begin{array}{l}\text { - No overlap between masters bus access } \\
\text { - Early stage in design }\end{array}$ & TLM \\
\hline - Main focus on application finish time & ATLM (b) \\
\hline - Main focus on individual transfer delay & ATLM (a) \\
\hline $\begin{array}{l}\text { - Synthesizable } \\
\text { - Using propagation delay }\end{array}$ & BFM \\
\hline
\end{tabular}

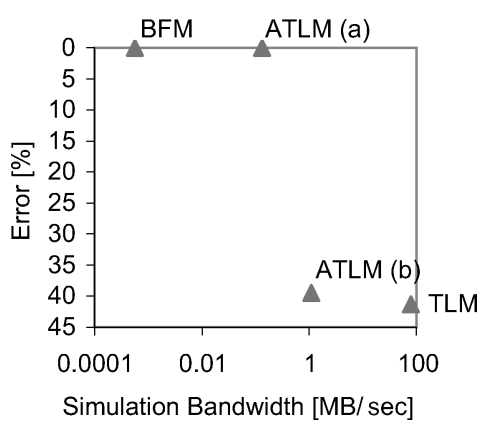

Fig. 19. CAN TLM trade-off.

\subsection{Summary for the CAN}

Based on our analysis, Table V lists the fastest model that yields acceptable results for a given situation and simulation focus.

The TLM can only be used in very early stages of the design. Its accuracy, for individual and cumulative transfer time, degrades drastically with increasing bus contention. The ATLM (b) is still fast. It is applicable in scenarios where the main focus is on the application finish time. However, this model is not suitable for predicting an individual transfer delay, since the duration-based analysis has not shown acceptable results.

The ATLM (a), which includes bit stuffing and CRC calculation, has shown $100 \%$ accuracy given the test restrictions (e.g., no propagation delay). It is the fastest model that accurately predicts the delay of an individual transfer in all contention situations. The BFM is necessary as a synthesizable model or in case the simulation includes propagation delay on the simulated CAN bus.

Figure 19 depicts the TLM trade-off for the CAN using the average individual timing error of the lower priority node. The accurate models BFM and ATLM (a) are slow with a bandwidth of less than $1 \mathrm{MB} / \mathrm{sec}$. The faster models yield inaccurate results, the ATLM (b) with $40 \%$ error. The fastest model, the TLM with close to $100 \mathrm{MB} / \mathrm{sec}$, is also the most inaccurate with $43 \%$ error.

We classify the CAN as an example for an off-chip serial bus with decentralized arbitration. Its results can be used as an indicator for modeling other serial bus systems, such as $\mathrm{I}^{2} \mathrm{C}$ [Philips 2000] and Ethernet.

\section{COLDFIRE MASTER BUS}

Our third bus example, the ColdFire Master Bus (Version 2.0) [Motorola 1997], falls into the category of custom CPU busses. Typically, those busses are simpler 


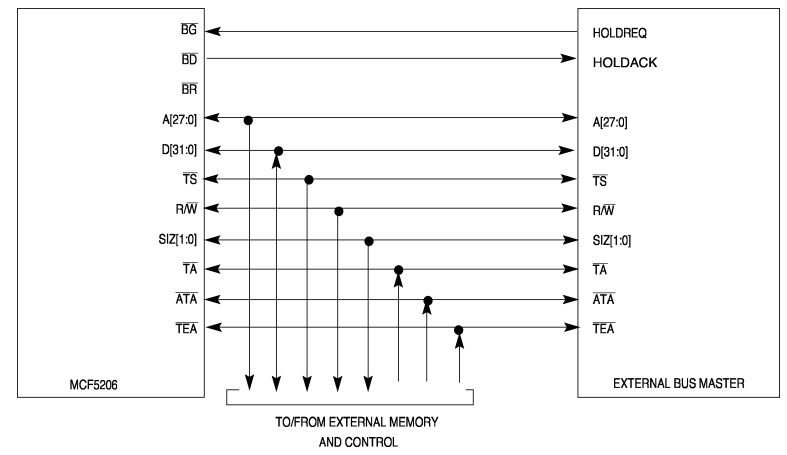

Fig. 20. ColdFire Master bus with two masters (Source: Motorola [1997]).

than general standard busses, since they have been designed for the specific requirements of one embedded processor.

\subsection{Introduction}

Motorola (now Freescale Semiconductor, Inc.) has introduced the ColdFire Master Bus for its popular microprocessor ColdFire MCF5206. It is a 32-bit tristate bus that uses basic transfers (byte, short, word), as well as a 4 beat burst to fill or store a cache line. It has to be noted that the line access (like a 4-beat burst) cannot be preempted. Therefore, there is no distinction between locked and unlocked transfers, as we have seen for the AMBA AHB.

The ColdFire Master Bus allows the usage of two arbitration schemes. One, the three wire mode, involves an external arbiter and allows a true multimaster operation. The second option, the two wire mode, connects two masters that directly hand over the bus without the need of an external arbiter, as shown in Figure 20. We have selected the operation mode with two masters to complement the centralized arbitration scheme used for the AMBA AHB.

\subsection{Models}

Our modeling of the ColdFire Master bus follows our scheme of abstracting data and arbitration, as described in Section 3. Again, we have implemented three major models: the BFM that implements a bus cycle access, the ATLM with a granularity of bus primitives, and the user transaction based TLM. We have not implemented any variants of these models.

\subsection{Performance Analysis}

We have measured the performance of the ColdFire Master bus in the setup with one master and one slave as described in Section 4.1. Figure 21 shows the simulation bandwidth.

Again, the measurements confirm the performance increase with abstraction. Also, the characteristic saw tooth shape, as seen already for the AHB, repeats. Due to the split of user transactions into bus transactions, the number of bus transactions does not increase linearly with an increase in size. 


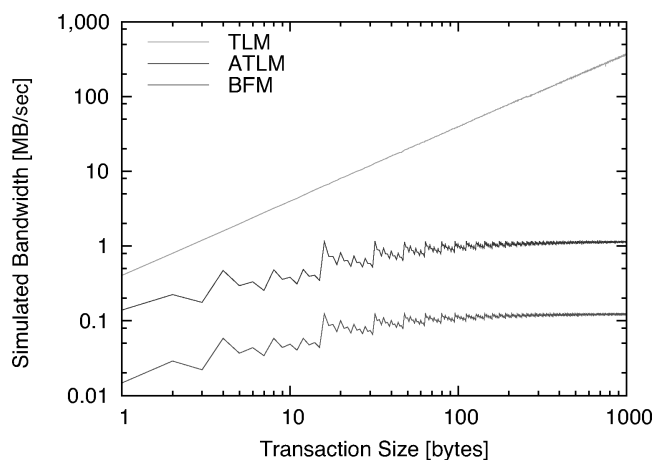

Fig. 21. Performance of the ColdFire Master bus models.

Table VI. Performance Comparison for Transferring 512 bytes Using ColdFire Master Bus Models

\begin{tabular}{|l||c|c|c|}
\hline & BFM & ATLM & TLM \\
\hline \hline Simulation Time $[\mathrm{ms}]$ & 3.93 & 0.423 & 0.0026 \\
\hline Sim. Bandwidth $[\mathrm{MB} / \mathrm{sec}]$ & 0.124 & 1.15 & 189.7 \\
\hline Speedup over BFM & 1.0 & 9.3 & 1525.0 \\
\hline Rel. Speedup over previous & - & 9.3 & 164.3 \\
\hline
\end{tabular}

The BFM shows the lowest simulation bandwidth, since it models each individual bus cycle. The ATLM is one order of magnitude faster, due to the abstraction to bus transactions. The TLM is the fastest model. By simulating whole user transactions, it surpasses the ATLM by two orders of magnitude. Table VI lists detailed numerical results for transferring 512 bytes.

\subsection{Accuracy Analysis}

We have analyzed the timing accuracy in the setup with two concurrent masters as described in Section 4.2. As for the AHB, each master sends 5,000 predefined user transactions that vary linear randomly in address, size ( $1 . .100$ bytes), and delay to the next transfer. Figure 22 depicts the accuracy of both masters over an increasing amount of bus contention.

As intended, the BFM complies with the standard and exhibits $0 \%$ error (its graph lies on top of the x-axis). The ATLM also reaches 100\% accuracy. Since the ColdFire Master bus does not support preemption, the arbitration handling at the boundary of the bus transaction is sufficient. In case the high-priority master requests the bus during an ongoing burst of the low-priority master, the high-priority master has to wait until the burst completes and the low priority master releases the bus. The bus ownership changes only at the boundary of a bus transaction. Thus, the ATLM, which models this granularity, is accurate and its graph lies on top of the x-axis.

The TLM exhibits a linear increase in error with increasing bus contention, since it models the transfers at the coarser granularity of user transactions. At $45 \%$ bus contention, the TLM shows 54\% error for the low-priority master. 


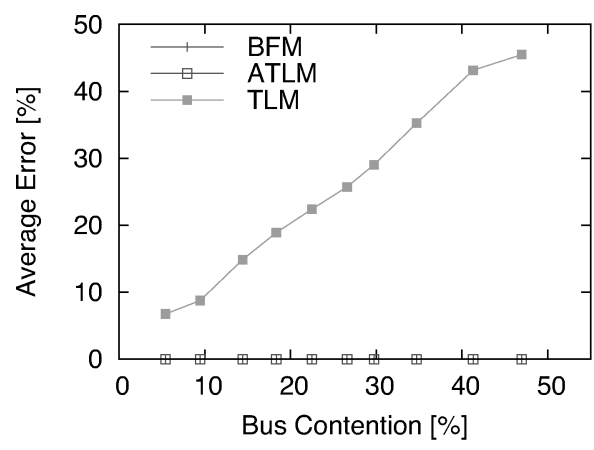

(a) High-priority master.

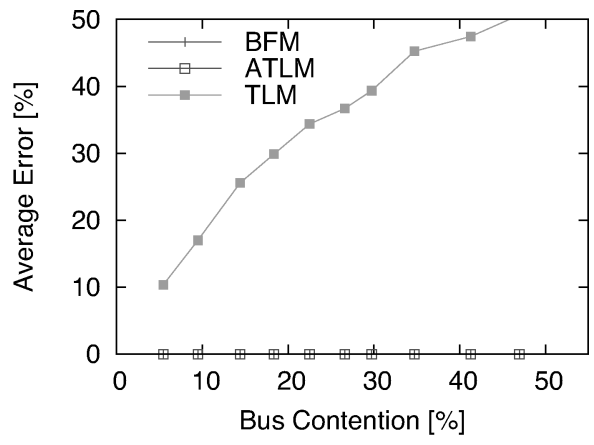

(b) Low-priority master.

Fig. 22. Individual timing accuracy for the ColdFire Master bus models.

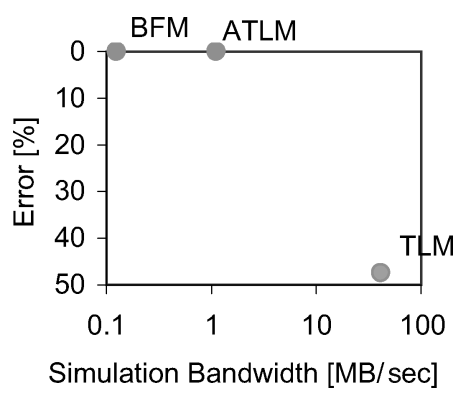

Fig. 23. ColdFire Master bus TLM trade-off.

Analyzing the cumulative transfer times yields very similar results, so we omit these graphs. The TLM is the only model that exhibits an error, which reaches $35 \%$ at $45 \%$ bus contention.

\subsection{Summary for the ColdFire Master Bus}

With the Motorola ColdFire Master bus being simpler than the other protocols, its speedup with abstraction is not as dramatic. The most abstract TLM is 1,525 times faster than the BFM. At the same time, the TLM is the only model that produces inaccurate results, with up to 54\% error. The ATLM already simulates accurately, since the bus does not support burst preemption. However, the ATLM is only 9.3 times faster than the BFM.

Figure 23 shows the trade-off for the ColdFire Master bus. The accurate models BFM and ATLM are slow with a bandwidth below $1 \mathrm{MB} / \mathrm{sec}$. The fast TLM, on the other hand, only yields inaccurate results with an average $47 \%$ error.

\section{GENERALIZATION}

Combining the performance and accuracy results of the three modeled bus systems, we will now generalize this data to give a broader perspective on the benefits and drawbacks of TLM. While, for an existing multitude of embedded 
Table VII. Speedup Over Bus Functional Model

\begin{tabular}{|l||c|c|c|}
\hline Bus & ATLM (a) & ATLM (b) & TLM \\
\hline \hline AMBA AHB & 78 & 81 & 6802 \\
\hline CAN & 228 & 1879 & 22124 \\
\hline ColdFire & 9.3 & - & 1525 \\
\hline
\end{tabular}

bus architectures, an extrapolation based on three individual models is difficult in general, our data is a strong indication of generality.

As basis for our generalization, we have carefully selected three standard examples found in a majority of real-world embedded systems that exhibit very diverse communication properties. The AMBA AHB is a general-purpose onchip pipelined parallel bus with central arbitration and a multiplexed interconnection scheme. In contrast, the CAN is a dedicated off-chip serial bus with distributed arbitration and a dominant-recessive interconnection. Yet, a different candidate is the Motorola ColdFire Master Bus, a special-purpose embedded processor bus with custom arbitration and tristate interconnection. Thus, these three chosen busses cover a wide variety of bus categories and can therefore serve as basis for a generalization with strong confidence.

Moreover, our granularity-based modeling approach is general and applicable to any layer-based bus model. Finally, we have observed the very same performance and accuracy patterns in our analysis for all three cases.

\subsection{Performance}

All our measurements confirm that TLM abstraction dramatically increases simulation performance (Table VII). Decreasing granularity of user data handling and arbitration is a very efficient method of abstraction. For all models, we have measured a speedup of orders of magnitude with each step of coarser granularity. The cycle-accurate BFM performs slowly for all busses. The ATLM, that simulates on a bus transaction granularity, is at least one order of magnitude faster. Further decreasing the granularity to user transactions, as done by the TLM, increases the speed by another two orders of magnitude.

On the other hand, variants at the same granularity level do not yield any significant speedup. We see this clearly in the ATLM variants for the AMBA AHB. In other words, models within the same granularity category simulate at the same speed. It should be noted that we have observed the same fact also for variants at the TLM abstraction in previous work [Schirner and Dömer 2006].

Only, if a feature requires a significant computation effort, then abstracting even at the same granularity level can improve performance. As an example, the ATLM (b) of the CAN does not model bit inspection and simulates 8 times faster than its counterpart at the same level.

Our analysis also shows that the potential performance gain for a model depends on the complexity of the actual protocol. We have achieved the highest speedup of 22,124× over the BFM for the complex CAN protocol. On the other hand, for the relative simple protocol of the custom CPU bus, the Motorola ColdFire Master bus, the TLM is only 1525 times faster. 
Table VIII. Average Individual Timing Error for the Low-Priority Master at $40 \%$ Bus Contention

\begin{tabular}{|l||c|c|c|c|}
\hline Bus & BFM & ATLM (a) & ATLM (b) & TLM \\
\hline \hline AMBA AHB (locked) & $0 \%$ & $0 \%$ & $18 \%$ & $32 \%$ \\
\hline AMBA AHB (unlocked) & $0 \%$ & $31 \%$ & $31 \%$ & $37 \%$ \\
\hline CAN & $0 \%$ & $0 \%$ & $39 \%$ & $42 \%$ \\
\hline ColdFire & $0 \%$ & $0 \%$ & - & $47 \%$ \\
\hline
\end{tabular}

\subsection{Accuracy}

Table VIII summarizes the results of our accuracy analysis. The accuracy achieved by a model depends on the modeling granularity and the actual granularity of the bus. By definition, each BFM is accurate, since it is the most fine-grained model. The ATLMs based on bus transactions are accurate if and only if the modeling granularity matches the actual granularity of the bus. The ATLM (a) for the AHB was accurate in the locked transfer mode as well as the ATLMs for the CAN and the ColdFire Master bus. Here, the actual bus protocol arbitrates once per bus transaction.

Inaccuracy has to be accepted when the model is more coarse grained. The ATLM (a) is inaccurate in the unlocked mode of the AMBA AHB, since bursts can be preempted and an arbitration decision within a bus transaction would be necessary. Furthermore, all our TLMs are inaccurate, due to the high abstraction.

Abstracting away timing relevant features also leads to an inaccurate model, as shown for the CAN ATLM (b) that omits CRC calculation and bit stuffing. Also, the abbreviated arbitration of the ATLM (b) of the AMBA AHB yields an inaccurate model.

Finally, the actual measured inaccuracy of a model highly depends on the bus contention. The error of coarse grain arbitration modeling only takes effect if two masters access the bus simultaneously. In other words, all our models are accurate if there is no bus contention. Therefore, the most abstract model is the best choice if the architecture at hand only uses a single master, or if a very low bus contention can be expected. On the same note, modeling point-to-point dedicated links that inherently do not have any bus contention, can also be accurately modeled at the highest TLM abstraction.

\subsection{TLM Trade-Off}

Figure 24 summarizes our measurements and depicts the TLM trade-off for all our bus models. On the x-axis, Figure 24 denotes the simulation performance in terms of the simulation time for a 100 byte user transaction. The y-axis denotes the average error of the model for the low-priority master at $40 \%$ bus contention.

Abstract modeling poses a trade-off, with either fast or accurate results. The accurate BFMs are slow with less than $0.2 \mathrm{MB} / \mathrm{sec}$ bandwidth. On the other hand, the fast TLMs with a bandwidth of up to $100 \mathrm{MB} / \mathrm{sec}$ produce an average error from $32 \%$ to $47 \%$. 


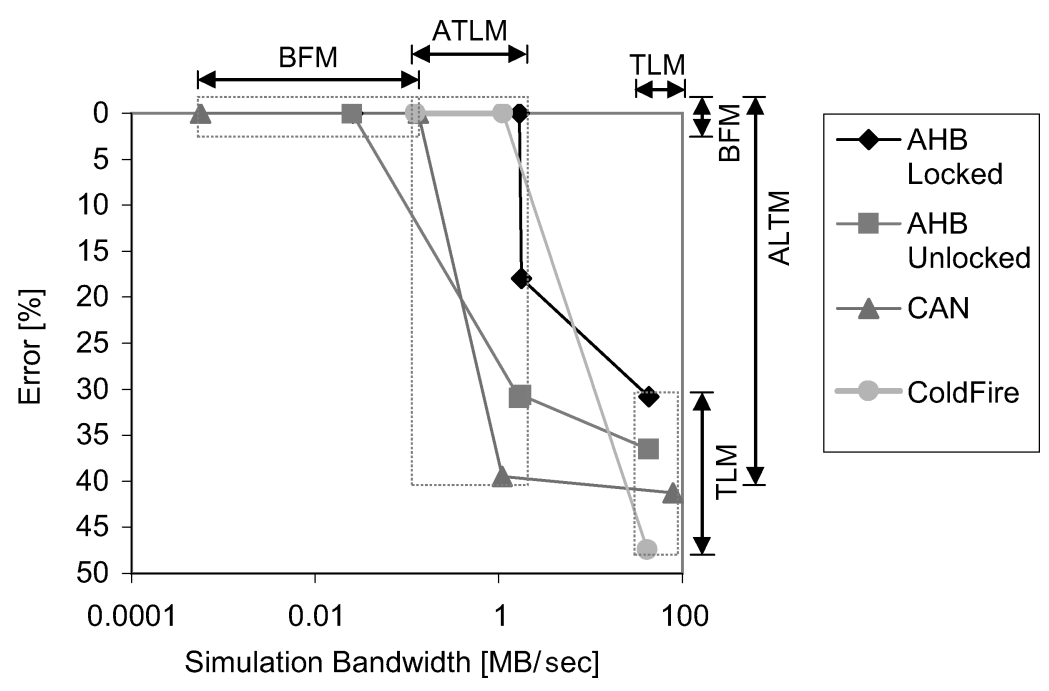

Fig. 24. TLM trade-off summary.

The ATLMs are found in the middle, simulating at about $1 \mathrm{MB} / \mathrm{sec}$ bandwidth. Some are accurate, since the modeled granularity matches the granularity of the actual protocol. If the granularity does not match, or in case of additional feature abstraction, the ATLMs generate an average error from $18 \%$ to $39 \%$.

\section{CONCLUSIONS}

Our contributions in this article are fourfold. First, we have classified TLM according to the granularity of data and arbitration handling. Second, we have defined appropriate metrics and setups for a systematic quantitative analysis of TLM with respect to simulation performance and timing accuracy. Third, we have quantified the TLM trade-off for three diverse communication protocols. Fourth, we have generalized our observations from the analysis to guide communication model designers and model users.

In particular, we have applied our granularity-based abstraction to three common bus systems covering diverse communication protocols: first the AMBA $\mathrm{AHB}$, as an on-chip parallel bus with centralized arbitration, second the CAN, as an off-chip decentralized serial bus system, and third the ColdFire Master bus, as a custom embedded processor bus. We have modeled, validated, and systematically analyzed each bus using our performance and accuracy metrics.

Based on the analysis results of the individual examples, we have then derived general conclusions. Abstraction based on a decreasing (coarsening) granularity yields at least an order of magnitude improvement per granularity level. On the other hand, abstracting features at the same granularity level only yields marginal performance improvements. In other words, proper model granularity is the key to efficient TLM abstraction.

However, TLM abstraction results in a serious loss in accuracy if the modeled granularity is more coarse grain than the granularity present in the actual bus protocol. This defines the TLM trade-off. In general, a model is either fast or 
accurate. Our fast TLM models with up to $100 \mathrm{MB} / \mathrm{sec}$ bandwidth show an error of up to $47 \%$. Accurate models, on the other hand, are slow. Our BFMs simulate with less than $0.2 \mathrm{MB} / \mathrm{sec}$ bandwidth.

The actual measured timing error highly depends on the bus contention. All our models are accurate in the absence of bus contention. Therefore, in the special case of a single master architecture, the most abstract TLM can be used without loss of accuracy.

In conclusion, this article contributes a systematic quantitative analysis of performance and accuracy in TLM, using a diverse set of major bus architecture standards, that confirms the TLM promise of high simulation speed. Our detailed analysis also identifies conditions for abstract and accurate models. As a result, we provide guidelines to the designer of communication models for efficiently abstracting communication protocols. The same guidelines also allow the system designer as a user of communication models to make an informed decision about the appropriate model for the design at hand.

\section{REFERENCES}

ARM. 1999. AMBA Specification (Rev. 2.0), ARM IHI 0011A. Advanced RISC Machines Ltd. (ARM).

ARM. 2003. AMBA AHB Cycle Level Interface (AHB CLI) Specification, ARM IHI 0011A. Advanced RISC Machines Ltd (ARM).

Bosch. 1991. CAN Specification, 2.0 Ed. Robert Bosch GmbH.

BREM, D. AND MülLER, D. 2003. Interface based system modeling of a can using sve. In Proceedings of the EkompaSS Workshop.

CAI, L. AND GAJSkI, D. 2003. Transaction level modeling: An overview. In Proceedings of the International Conference on Hardware/Software Codesign and System Synthesis.

Caldari, M., Conti, M., Coppola, M., Curaba, S., Pieralisi, L., and Turchetti, C. 2003. Transactionlevel models for AMBA bus architecture using SystemC 2.0. In Proceedings of the Design, Automation and Test in Europe (DATE) Conference.

Coppola, M., Curaba, S., Grammatikakis, M., and Maruccia, G. 2003. IPSiM: SystemC 3.0 enhancements for communication refinement. In Proceedings of the Design, Automation and Test in Europe (DATE) Conference.

Gajski, D. D., Zhu, J., Dömer, R., Gerstlauer, A., and Zhao, S. 2000. SpecC: Specification Language and Design Methodology. Kluwer Academic Publishers.

Gasteier, M. And Glesner, M. 1996. Bus-based communication synthesis on system-level. In Proceedings of the International Symposium on System Synthesis.

Gerstlauer, A. ANd GajSKi, D. D. 2002. System-level abstraction semantics. In Proceedings of the International Symposium on System Synthesis.

Gerstlauer, A., Shin, D., Doemer, R., And Gajski, D. 2005. System-level communication modeling for network-on-chip synthesis. In Proceedings of the Asia and South Pacific Design Automation Conference.

Gerstlauer, A., Shin, D., Peng, J., Dömer, R., and Gajski, D. D. 2007. Automatic layer-based generation of system-on-chip bus communication models. IEEE Trans. Comput. Aid. Design Intergr. Circ. Syst. 26, 9, 1676-1687.

Grötker, T., Liao, S., Martin, G., And Swan, S. 2002. System Design with SystemC. Kluwer Academic Publishers.

Hartwich, F. and Bassemir, A. 1999. The Configuration of the CAN Bit Timing. Robert Bosch GmbH. http://www. can. bosch.com.

Haverinen, A., LeclercQ, M., Weyrich, N., and Wingard, D. 2002. SystemC based SoC communication modeling for the OCP protocol. http://www .ocpip.org.

IBM. 2004. 128-bit processor local bus architecture specification, SA-14-2538-04, 4.6 Ed. IBM.

ISO. 1994. Reference model of open system interconnection (OSI) 2nd Ed. Internation Organization for Standardization (ISO). ISO/IEC 7498 Standard.

ACM Transactions on Embedded Computing Systems, Vol. 8, No. 1, Article 4, Publication date: December 2008. 
Lahiri, K., Raghunathan, A., ANd Dey, S. 2001. System-level performance analysis for designing on-chip communication architectures. In IEEE Trans. Comput. Aid. Design Intergr. Circ. Syst. 20. 768-783.

Lajolo, M., Passerone, C., and Lavagno, L. 2003. Scalable techniques for system-level cosimulation and co-estimation. IEE Proc. 150, 4, 227-238.

Motorola 1997. MCF5206 ColdFire Integrated Microprocessor User's Manual. Motorola.

Pasricha, S., Dutt, N., and Ben-Romdhane, M. 2004. Fast exploration of bus-based on-chip communication architectures. In Proceedings of the International Conference on Hardware / Software Codesign and System Synthesis (CODES / ISSS).

Philips. P8xC592: 8-bit microcontroller with on-chip CAN. Philips.

Philips. 2000. The $\mathrm{I}^{2} \mathrm{C}$-bus specification, $2.1 \mathrm{ed}$. Philips.

Rose, A., Swan, S., Pierce, J., And Fernandez, J.-M. 2005. Transaction level modeling in SystemC. http: //www. systemc.org.

Sarmento, A., Cesario, W., and Jerraya, A. A. 2001. Mixed-level cosimulation for fine gradual refinement of communication in SoC design. In Proceedings of the Design, Automation and Test in Europe (DATE) Conference.

SchiRner, G. AND Dömer, R. 2005a. Abstract communication modeling: A case study using the CAN automotive bus. In From Specification to Embedded Systems Application, A. Rettberg, M. Zanella, and F. Rammig, Eds. Springer.

Schirner, G. AND Dömer, R. 2005b. System level modeling of an AMBA bus. Tech. rep. CECSTR-05-03, Center for Embedded Computer Systems, University of California, Irvine.

Schirner, G. AND Dömer, R. 2006. Quantitative analysis of transaction level models for the AMBA bus. In Proceedings of the Design, Automation and Test in Europe (DATE) Conference.

Sgroi, M., Sheets, M., Minal, M., Keutzer, K., Malik, S., Rabaey, J., , and Sangiovanni-Vincenteldi, A. 2001. Addressing the system-on-a-chip interconnect woes through communication based design. In Proceedings of the Design Automation Conference.

Siegmund, R. ANd Müller, D. 2001. SystemC ${ }^{S V}$ : An extension of SystemC for mixed multilevel communication modeling and interface-based system design. In Proceedings of the Design, Automation and Test in Europe (DATE) Conference.

Received June 2006; revised February 2007; accepted August 2007 